\title{
DEVELOPMENT OF RIGOROUS METHODS IN FLUID MECHANICS AND THEORY OF WATER WAVES
}

\author{
ANDREI ERMAKOV D
}

(Received 24 May 2020; first published online 11 September 2020)

2020 Mathematics subject classification: primary 76B25; secondary 00A69, 35C08, 76B15.

Keywords and phrases: water waves, wave scattering, solitary wave, forced Korteweg-de Vries equation.

The transformation of surface waves over varying obstacles is far from a new problem, but it has become an increasing challenge in our world due to growing environmental, societal and technological needs. Many stakeholders, from scientists and engineers through to governments and private companies, are searching for superior models to describe the influences of changes in depth of the ocean or cross-section of canals, and how the speed of the current affects the wave transformation. This research can have many impacts, from protecting coastlines or natural treasures like the Great Barrier Reef and bringing greater safety to marine vessels and offshore structures, to answering complex new theories and concepts, including the application of the Hawking effect in relation to black holes. Whenever a wave passes over a surface, the size of the transmitted wave may be significantly increased compared to the amplitude and speed of the incident wave. The impact of a transmitted wave on offshore or coastal structures is impossible to calculate without closely calculating the transmitted waves. It is also impossible to trust the numerical results without deep analytical modelling of these problems due to the complex nature of the effects. This research looks at four important considerations: the transformation of long linear waves in an ocean with a variable depth, long-wave scattering in a canal with a rapidly varying cross-section, long linear surface waves on stationary currents in a canal of constant depth and variable width, and the re-estimation of the forced Korteweg-de Vries equation to describe a resonant interaction of a solitary wave with external pulse-type perturbations.

Chapter 1 reviews the history of these problems and notes previous literature and research in this area.

In Chapter 2, we calculate the transformation of long linear waves in an ocean of variable depth. Here, the transformation coefficients (transmission and reflection) are

Thesis submitted to the University of Southern Queensland in September 2019; degree approved on 30 March 2020; supervisors Yury Stepanyants and Dmitry Strunin.

(C) 2020 Australian Mathematical Publishing Association Inc. 
considered as functions of frequency and the total depth drop for three typical models of bottom profile variation: piecewise-linear, piecewise-quadratic and hyperbolic tangent profiles. We compare the influence of the different bottom profiles on the wave transformation.

In Chapter 3, we consider long-wave scattering in a canal with a rapidly varying cross-section, where the scattering coefficients (transmission and reflection) are calculated for all possible orientations of the incident wave, including background current (downstream and upstream propagation) and current type (subcritical, transcritical and supercritical). Sometimes, when over-reflection or over-transmission occurs, negative-energy waves can appear. A spontaneous wave generation can happen in a transcritical accelerating flow, when canal narrowing increases the background current. This resembles spontaneous wave generation on the horizon of an evaporating black hole due to the Hawking effect.

In Chapter 4, we study long linear surface waves on stationary currents in a canal of constant depth and variable width. Here, we consider both the co-current and counter-current wave propagation in accelerating and decelerating currents and calculate the scattering coefficients as functions of incident wave frequency for all possible cases of sub-, super- and transcritical currents. Results are analysed in application to recent analogue gravity experiments which shed light on the problem of hydrodynamic modelling of Hawking radiation, as published in [2].

In Chapter 5, we revise the solutions of the forced Korteweg-de Vries equation by describing a resonant interaction of a solitary wave with external pulse-type perturbations (nonlinear ocean). Unlike previously narrower works, we create an arbitrary relationship between the widths of a soliton and an external perturbation of a relatively small amplitude. In many cases, exact solutions of the forced Korteweg-de Vries equation can be obtained for the specific forcings of arbitrary amplitude. The earlier developed asymptotic method is used to gain an approximate set of equations up to the second order in a small parameter characterising the amplitude of external force. The theoretical outcomes obtained by the asymptotic method are in good agreement with the results of direct numerical modelling within the framework of the forced Korteweg-de Vries equation. In the Conclusion, we observe the results gained from various models and equations and discuss the various applications, innovations and future applications of these results, as well as what changes and what problems need to be considered for future research.

Some of the work from the thesis has been published in [1-4].

\section{Acknowledgments}

This research and subsequent publications would not have been possible without the generous support of the University of Southern Queensland's Research Training Program, in partnership with the Australian Government. 


\section{References}

[1] S. M. Churilov, A. M. Ermakov, G. Rousseaux and Y. A. Stepanyants, 'Scattering of long water waves in a canal with rapidly varying cross-section in the presence of a current', Phys. Rev. Fluids 2(9) (2017), Article ID 094805.

[2] S. M. Churilov, A. M. Ermakov and Y. A. Stepanyants, 'Wave scattering in spatially inhomogeneous currents', Phys. Rev. D 96(6) (2017), Article ID 064016.

[3] A. M. Ermakov and Y. A. Stepanyants, 'Soliton interaction with external forcing within the Korteweg-de Vries equation', Chaos 29(1) (2019), Article ID 013117.

[4] A. M. Ermakov and Y. A. Stepanyants, 'Transformation of long surface and tsunami-like waves in the ocean with a variable bathymetry', Pure Appl. Geophys. 177 (2020), 1675-1693.

\footnotetext{
ANDREI ERMAKOV, Centre for Ocean Energy Research, Maynooth University, Maynooth, Co. Kildare, Ireland

e-mail: ermakovandreim@gmail.com
} 